\title{
A new mathematical model to cover crew pairing and rostering problems simultaneously
}

\author{
Saeed Saemi*, Alireza Rashidi Komijan**, Reza Tavakkoli-Moghaddam*** and Mohammad Fallah* \\ *Dep. of Industrial Engineering, Central Tehran Branch, Islamic Azad University, Tehran, Iran \\ **Dep. of Industrial Engineering, Firoozkooh Branch, Islamic Azad University, Firoozkooh, Iran \\ ***School of Industrial Engineering, College of Engineering, University of Tehran, Tehran, Iran \\ **Corresponding Author: rashidi@azad.ac.ir
}

Submitted: 28/09/2019

Revised: $06 / 04 / 2020$

Accepted: $08 / 07 / 2020$

\begin{abstract}
Crew scheduling problem includes two separate subproblems, namely, crew pairing and crew rostering problems. Solving these two subproblems in a sequential order may not lead to an optimal solution. This study includes two main novelties. It combines these two subproblems and presents them in a single model. On the other hand, despite previous researches that considered a pairing continuously, the proposed model benefits from the capability of considering one or more days off in a pairing assigned to a crew member. This is extremely useful as it enables the crew to participate in required courses, doing medical checks, etc. Two solution approaches, namely, Genetic Algorithm (GA) and Particle Swarm Optimization (PSO), are used to solve the model. Eventually, the performance of the proposed algorithms is evaluated. Both ended to satisfactory results; however, PSO relatively outperformed GA in terms of solution optimality and computational time.
\end{abstract}

Keywords: Crew Scheduling; Crew Pairing; Crew Rostering; Meta-Heuristics; Genetic Algorithm; Particle Swarm Optimization.

\section{INTRODUCTION}

Operations research techniques have been used in the air industry since the 1950s (Barnhart and Talluri, 1997). These techniques result in a reduction of operational costs and, consequently, an increase in revenue in this competitive industry. Generally, airline scheduling includes flowing stages:

$\checkmark$ Flight Scheduling Problem (FSP)

$\checkmark$ Fleet Assignment Problem (FAP)

$\checkmark$ Aircraft Maintenance Routing Problem (AMRP)

$\checkmark$ Crew Scheduling Problem (CSP)

FSP is the basis of operational planning in airlines. In this problem, the origin and destination, as well as the departure and arrival time of flights, are determined. The first phase aims to offer certain flights; consequently, some factors such as market demand, aviation rules, workforce regulations, and the behavior of rivals should be considered (Bazargan, 2016). The second phase in airline scheduling is to assign different aircraft types to the flights scheduled throughout the previous step. This problem is called FAP and is highly affected by the expected flight demands. Higher flight demands need larger aircrafts, and smaller ones need smaller aircrafts (Dong et al., 2016). In AMRP, a set of sequential flights is assigned to each aircraft, while periodic maintenance requirements and other restrictions of 
federal aviation administration (FAA) are taken into account (Haouari et al., 2013). The final stage is crew (cockpit/ cabin) scheduling. Crew cost is the second significant cost after fuel expenditures in airlines. Therefore, even a small improvement may have a noticeable influence on airline outcomes (Graves et al., 1993). CSP includes two subproblems: crew pairing and rostering problems (CPP and CRP). In the first subproblem, all pairings are generated and selected according to the aim of cost minimization. Moreover, some FAA and resource human regulations should be considered as well (Ahmadbeygi et al., 2009).

In CRP, the pairings generated in the previous subproblem are assigned to the crew members while considering educational courses, vacations, and so on. Rostering should be planned one month before the day of operation. Moreover, some complex rules should be considered in the rostering problem (Kohl and Karisch, 2004). Due to unexpected events, some flights may be uncovered. Therefore, a set of reserved crew members are needed to be used in such situations to cover all the flights (Sohoni et al., 2006).

In this paper, a new formulation for integrated CSP is presented. Some advantages of this formulation are as follows:

1. Solving CPP and CRP in a sequential order may result in suboptimal solutions. Hence, in this paper, an integrated model is presented using a new formulation.

2. The model can determine the best number of crew members that need to be assigned; therefore, some of them can be idle and used as reserved crew members for avoiding delays and cancellations due to unexpected events during the day of operation.

3. The model can consider one or more days off in a pairing enabling crew to participate in required courses, doing medical checks, etc.

It should be noted that the process of assigning cockpit and cabin crew members is different. This paper focuses on cockpit crew members scheduling. The paper is organized as follows:

In Section 2, related literature is reviewed. Problem definition is presented in Section 3. In Section 4, a new model is formulated to integrate CPP and CRP considering the rules related to the FAA, some of which are mentioned in this Section. Section 5 includes the introduction of the applied metaheuristic algorithms. In Section 6, the comparison of the results obtained from the solution approaches is made. Finally, the results obtained from the integrated model are compared with those of sequential models is in Section 7.

\section{LITERATURE REVIEW}

$\mathrm{CPP}$ is generally formulated as a set covering problem (SCP) or a set partitioning problem (SPP). Ahmadbeygi et al. (2009) proposed a model to generate and select pairings and solved it using traditional solvers. They integrated the column generation with the branch and bound algorithm to solve the model. The results on the real data set related to the US airway showed good performance of their approach. Zeren and Ozkol (2012) introduced a new genetic operator to solve CPP and applied it to Turkish airline. The results showed that the new operator dramatically improved the convergence rate. Aydemir-Karadag et al. (2013) used SCP to formulate CPP and introduced three algorithms to solve it. The comparison of the randomly generated data set problems confirmed the better function of the last two algorithms compared to the first one. Zeren and Ozkol (2016) presented a new column generation strategy for solving CPP in large-scale problems. The results showed that the new strategy has a number of advantages, such as improvement in computational time. Quesnel et al. (2017) addressed the extension of CPP by considering some additional constraints to each crew base. These constraints limited the total work time and provided a better solution to solve the CRP. They introduced a new branching scheme based on column generation. Some advantages of this new approach were more optimal solutions in comparison with the traditional methods. Deveci and Demirel (2018) solved CPP using Genetic and Memetic Algorithms and compared their efficiencies. The results showed higher efficiency of the employed MA compared to GA. Haouari et al. (2019) presented a new formulation for CPP. This model was nonlinear mixed integer programming that could be solved in polynomial time. They linearized the model and compared the enhanced model 
with the linearized one. The results demonstrated that the enhanced model could be used in airlines without using complicated algorithms. Ionescu and Kliewer (2011) proposed a new stochastic robust formulation for CPP. They provided swap opportunities for the crew during duty days. They solved the model using column generation. Muter et al. (2013) presented a new formulation for CPP in such a way that some extra flights could be inserted in the duty day. They used column generation as their solution approach.

A large number of researches have been done in the field of CRP. Santosa et al. (2010) used the Differential Evaluation (DE) algorithm to solve CRP and finally compared it with the results of the column generation method in different numerical examples. Moreover, Hadianti et al. (2013) proposed a new nonlinear integer model and solved it using the Simulated Annealing (SA) algorithm. The results showed that this approach was well-defined for the problem and could achieve good solutions over a short time. Chen and Chou (2016) presented a new multiobjective formulation for crew rostering recovery problem (CRRP) and solved it using NSGA-II algorithm. De Armas et al. (2017) presented a new multistart randomized heuristic algorithm to solve the real-life CRP. The objective function aimed to balance the workload between the crew members. Doi et al. (2018) solved CRP with a two-level decomposition algorithm. This algorithm decomposed the original problem into master and subproblems. They also proposed a new method to solve the master problem. The obtained results on the real data showed a higher efficiency of the proposed method. Gomes and Gualda (2015) presented a new constructive heuristic algorithm that generated integer solutions during a reasonable time for the problems of CSP with small and medium sizes. The results showed superiority in comparison to the previous results. Kasirzadeh et al. (2017) addressed the problem of CSP using a sequential procedure. They proposed new formulations for both of the subproblems and solved them employing column generation. Deng and Lin (2011) formulated CSP based on the Travelling Salesman Problem (TSP) and used the Ant Colony Optimization (ACO) algorithm as the solution approach. Azadeh et al. (2013) used Particle Swarm Optimization (PSO) algorithm and enhanced it by taking advantage of local search heuristic method. They compared the applied solution approach with Ant Colony Optimization and Genetic Algorithm. The results showed that PSO ends to better solutions compared to GA and ACO.

In some researches, CSP has been integrated into other airline scheduling problems. Some papers addressed the integration of CPP and AMRP (Cordeau et al., 2001; Mercier et al., 2005; Mercier and Soumis, 2007; Weide et al., 2010; Diaz-Ramirezet al., 2014; Ahmed et al., 2018). Sandhu and Klabjan (2007) proposed a new model for the integration of FA and CP problems. They applied column generation and Benders decomposition method to solve the problem. Gao et al. (2009) introduced a new MIP formulation for integrated FAP and CSP. Ozener et al. (2017) discussed the integration of FA and CP problems and used an optimization-based algorithm as a solution tool. The results admitted the efficiency and adequacy of this algorithm for large-scale problems. Moreover, some researchers focused on integrating of FA, AMR, and CP problems (Cacchiani and Salazar-Gonzalez, 2013; Salazar-Gonzalez, 2014). Ozdemir and Uskudar (2020) applied fuzzy Multi Criteria Decision Making (MCDM) method to determine the future strategies of a transportation system in Turkey. Interval type-2 TOPSIS, AHP methods, Balanced Score Card (BSC) framework, and the group decision-making technique were combined for making better decisions. The results indicated that the selected alternatives were in agreement with the experts' opinions.

\section{PROBLEM DEFINITION}

Crew Scheduling Problem aims to minimize the cost of assigning crew members to flights. The assignment is done considering some human work regulations and FAA policies. This problem includes two subproblems called Crew Pairing Problem (CPP) and Crew Rostering Problem (CRP). In CPP, a set of duty sequences (called pairings) are generated with the aim of cost minimization so that all flights are covered. It should be stated that each of the duties consists of a sequence of flights. In CRP, the pairings generated through CPP are assigned to the crew members considering educational course requirements, vacations, and so on. This paper has two main contributions. Firstly, crew pairing and rostering problems are integrated and presented in a single model. Decision variables of the model are defined in such a way that can address sequence of flights, as well as crew assignment. Secondly, the proposed model benefits from the capability of considering one or more days off in a single pairing. This feature has not 
been considered in previous researches. In all researches, a pairing included consecutive duty days. Relaxing this assumption enables the crew to participate in required courses and get medical checks. The departure and arrival times are predetermined inputs of CSP. Each crew member has a particular home base. Each crew member may be assigned several duties. Each crew member must start his/her first duty by a flight from his/her home base. During each duty, each crew member handles sequences of flights so that the destination of one flight is the origin of the other one. Following FAA rules, it is necessary to consider a minimum sit time for crew between consecutive flights on each duty. At the end of a duty, each crew member finishes his/her duty by a flight to a place where he/she rests for a night. If this place is not the same as the crew member's base, the hoteling cost should be considered. It is possible for the crew member to stay in that place for several days between his/her duties. It is incumbent upon the crew member to start his/her next duty by a flight from the station of the previous duty. It should be noted that if there are two consecutive duties on consecutive days, a sufficient night rest (called min rest) should be considered for the crew member. There is a possibility of covering a flight with more than one crew member (the concept of deadhead). In this case, the extra crew members use that flight as passengers in order to handle the next assigned flights. Since this kind of crew displacement results in no income for the airline, a deadhead flight cost is considered in the objective function per each extra crew member. This paper also determines the best number of the assigned crew members so that all of the flights are covered. Therefore, some of them will remain idle and can be used as reserved to avoid delays or cancelations during the operation day. In the case of using each of the assigned crew members, a particular amount of cost will be added to the objective function. Following FAA rules, each crew member must handle at most some flights on a duty and over the planning horizon. Also, he/she must fly no more than a maximum amount of time on a duty and over the planning horizon. Moreover, there is a limitation for elapsed time on a duty. In the following section, a mathematical model is presented based on the problem definition mentioned above.

\section{PROBLEM FORMULATION}

In this section, a new mathematical model is presented for CSP. Sets, indices, parameters, and decision variables are as follows:

\section{Sets and indices}

$I:$ Set of all crew members

$i$ : Index of crew member $(i \in I)$

$J$ : Set of all cities

$j:$ Index of city $(j \in J)$

$D$ : Set of all days of planning horizon (Saturday to Friday)

$d:$ Index of day $(\mathrm{d} \in D)$

F: Set of all duties

$f:$ Index of duty ( $\mathrm{f} \in \mathrm{F}$ )

$N$ : Set of flight rounds. Suppose that a crew member starts his/her duty from city $A$ to $B$ and continues it by flight from $B$ to $C$. For the flight $(A, B), n$ equals one and for the flight $(B, C), n$ equals two

$\mathrm{n}$ : Index of flight round $(\mathrm{n} \in N)$

$o_{i}$ : Home base of crew member i

\section{Parameters}

$d e_{j j^{\prime} d}$ : Depatrure time of flight $j j^{\prime}$ (flight from city $\mathrm{j}$ to $\mathrm{j}$ ') on day $\mathrm{d}$

$l_{j j^{\prime} d}$ : Arrival time of flight $j j^{\prime}$ on day $\mathrm{d}$ 
min_sit:Minimum required time between two consecutive flights on a single duty day

min_rest: Minimum rest required time between two consecutive flights on consecutive days

upper 1: Maximum flying time per duty

upper 2: Maximum flying time in the planning horizon

upper3: Maximum elapsed time per duty

$e_{j j^{\prime} \mathrm{d}}$ : Deadhead cost of flight $j j^{\prime}$ on day d

$k_{j j^{\prime} \mathrm{d}}$ : The cost of uncovered flight jj' on day d

$h$ : Hoteling cost of crew member

$r_{i}$ : cost of using crew i

$M:$ A big number

\section{Decision variables}

$x_{i j j^{\prime} d n}$ : If the flight $j j^{\prime}$ on day $\mathrm{d}$ is assigned to crew member $\mathrm{i}$ in flight round $\mathrm{n}, 1$ otherwise 0

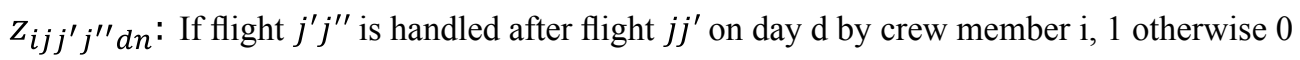

$s_{i j d}$ : If crew member i starts his/her duty on day d from city $\mathrm{j}, 1$ otherwise 0

$w_{i j d}$ : If crew member $\mathrm{i}$ ends his/her duty on day $\mathrm{d}$ to city $\mathrm{j}, 1$ otherwise 0

$y_{i d f}$ : If crew member i handles his/her f-th duty on day d, 1 otherwise $0(f \leq d)$

$S S_{i}$ : If crew member $i$ is assigned a duty in planning horizon, 1 otherwise 0

$v_{j j^{\prime} d}$ : If flight $j j^{\prime}$ on day d is covered, 1 otherwise 0

$b_{j j^{\prime} d}$ : Integer variable indicating the number of extra times that flight $j j^{\prime}$ is covered on day d (concept of deadhead)

The objective function and constraints of the model are as follows:

$$
\begin{aligned}
\min =\sum_{d \in D} \sum_{j^{\prime} \in J} \sum_{j \in J}\left(1-v_{j j^{\prime} d}\right) * k_{j j^{\prime} \mathrm{d}}+\sum_{d \in D} \sum_{j^{\prime} \in J} \sum_{j \in J}\left(b_{j j^{\prime} d}-v_{j j^{\prime} d}\right) * e_{j j^{\prime} d}+\sum_{d \in D} \sum_{j \neq o_{i}} \sum_{i \in I} w_{i j d} \\
* h+\sum_{i \in I} s s_{i} * r_{i}
\end{aligned}
$$

$\sum_{n \in N} x_{i j j^{\prime} d n} \leq 1 \quad \forall i \in I, j, j^{\prime} \in J, d \in D$

$\sum_{d \in D} \sum_{j^{\prime} \in J} \sum_{j \in J} x_{i j j^{\prime} d n} \leq 1 \quad \forall i \in I, n \in N$

$d e_{j^{\prime} j^{\prime \prime} d}-l_{j j^{\prime} d} \geq \min \_s i t-M *\left(2-x_{i j^{\prime} j^{\prime \prime} d, n+1}-x_{i j j^{\prime} d n}\right)$

$\forall i \in I, j, j^{\prime}, j^{\prime \prime} \in J, d \in D, n \in N$

$1440+d e_{j^{\prime} j^{\prime \prime} d+1}-l_{j j^{\prime} d} \geq \min \_r e s t-M *\left(2-x_{i j^{\prime} j^{\prime \prime} d+1, n+1}-x_{i j j^{\prime} d, n}\right)$

$$
\forall i \in I, j, j^{\prime}, j^{\prime \prime} \in J, d \in D, n \in N
$$




$$
\begin{aligned}
& s_{i j d} \geq-M *\left(1-y_{i d f}\right)+1 \quad \forall i \in I, j \in o_{i}, f \in F, f=1 \\
& w_{i j d} \geq 1-M *\left(1-y_{i d f}\right)-M *\left(\sum_{\substack{d^{\prime}=d+1 \\
d^{\prime} \in D}} \sum_{\substack{f^{\prime}=f+1 \\
f^{\prime} \in F}} y_{i d^{\prime} f^{\prime}}\right) \\
& \forall i \in I, j \in o_{i}, d \in D, f \in F: f \leq d
\end{aligned}
$$

$s_{i j d^{\prime}} \geq w_{i j d}-M *\left(2-y_{i d f}-y_{i d^{\prime} f+1}\right) \quad \forall i \in I, \quad j \in J, d, d^{\prime} \in D: d^{\prime}>d$

$s_{i j d^{\prime}} \leq w_{i j d}+M *\left(2-y_{i d f}-y_{i d^{\prime} f+1}\right) \quad \forall i \in I, j \in J, d, d^{\prime}, \in D: d^{\prime}>d$

$s_{i j d}+\sum_{n \in N} \sum_{j^{\prime} \in J} x_{i j^{\prime} j d}=\sum_{n \in N} \sum_{j^{\prime} \in J} x_{i j j^{\prime} d}+w_{i j d} \quad \forall i \in I, j \in J, d \in D$

$\sum_{n \in N} \sum_{j^{\prime} \in J} \sum_{j \in J} x_{i j j^{\prime} d n} \geq-M *\left(1-\sum_{f \in F} y_{i d f}\right)+\varepsilon \quad \forall i \in I, d \in D$

$\sum_{n \in N} \sum_{j^{\prime} \in J} \sum_{j \in J} x_{i j j^{\prime} d n} \leq M * \sum_{f \in F} y_{i d f} \quad \forall i \in I, d \in D$

$\sum_{f \in F} y_{i d f} \leq 1 \quad \forall i \in I, d \in D$

$\sum_{d \in D} y_{i d f} \leq 1 \quad \forall i \in I, f \in F$

$y_{i d f}=0 \quad \forall i \in I, d \in D, f \in F, d<f$

$M * S s_{i} \geq \sum_{d \in D} \sum_{f \in F} y_{i d f} \quad \forall i \in I$

$1-M *\left(2-y_{i d f}-x_{i j j^{\prime} d, n+1}\right) \leq \sum_{j^{\prime \prime} \in J} x_{i j^{\prime \prime} j d n}$

$\forall i \in I, j, j^{\prime} \in J, d \in D, n \in N, f \in F, f=1$

$\sum_{j^{\prime \prime} \in J} x_{i j^{\prime \prime} j d n} \leq 1+M *\left(2-y_{i d f}-x_{i j j^{\prime} d, n+1}\right)$

$\forall i \in I, j, j^{\prime} \in J, d \in D, n \in N, f \in F, f=1$

$1-M *\left(3-y_{i d^{\prime} f+1}-y_{i d f}-x_{i j j^{\prime} d^{\prime}, n+1}\right) \leq \sum_{j^{\prime \prime} \in J} x_{i j^{\prime \prime} j d^{\prime} n}+\sum_{j^{\prime \prime} \in J} x_{i j^{\prime \prime} j d n}$

$$
\forall i \in I, j, j^{\prime} \in J, d, d^{\prime} \in D: d^{\prime}>d, f \in F, n \in N
$$




$$
\begin{array}{r}
\sum_{j^{\prime \prime} \in J} x_{i j^{\prime \prime} j d^{\prime} n}+\sum_{j^{\prime \prime} \in J} x_{i j^{\prime \prime} j d n} \leq 1+M *\left(3-y_{i d^{\prime} f+1}-y_{i d f}-x_{i j j^{\prime} d^{\prime}, n+1}\right) \\
\forall i \in I, j, j^{\prime} \in J, d, d^{\prime} \in D: d^{\prime}>d, f \in F, n \in N
\end{array}
$$

$\sum_{j^{\prime} \in J} x_{i j j^{\prime} d n} \geq s_{i j d}-M *\left(1-y_{i d f}\right)$

$$
\forall i \in I, j \in J, d \in D, f \in F, f=1, n \in N, n=1
$$

$$
\begin{gathered}
\sum_{j^{\prime} \in J} x_{i j j^{\prime} d n} \geq s_{i j d}-M *\left(2-y_{i d^{\prime} f}-y_{i d f+1}\right)-M *\left(1-\sum_{j^{\prime \prime} \in J} \sum_{j^{\prime \prime \prime} \in J} x_{i j^{\prime \prime} j^{\prime \prime \prime} d^{\prime} n-1}\right) \\
-M *\left(\sum_{j^{\prime \prime} \in J} \sum_{j^{\prime \prime \prime} \in J} x_{i j^{\prime \prime} j^{\prime \prime \prime} d^{\prime} n}\right) \\
\forall i \in I, j \in J, d, d^{\prime} \in D: d^{\prime}<d, n \in N
\end{gathered}
$$

$$
\begin{gathered}
\sum_{d \in D} \sum_{j \in J} s_{i j d} \leq\left(d^{\prime \prime}-d^{\prime}+1\right)+M *\left(2-y_{i d^{\prime} f}-y_{i d^{\prime \prime} f^{\prime}}\right)+M *\left(\sum_{d^{\prime \prime \prime} \in D} y_{i d^{\prime \prime \prime}\left(f^{\prime}+1\right)}\right) \\
\forall i \in I, d^{\prime \prime}, d^{\prime} \in D, f^{\prime}, f \in F, f=1
\end{gathered}
$$

$\sum_{d \in D} \sum_{j \in J} s_{i j d} \leq M *\left(\sum_{d \in D} \sum_{f \in F} y_{i d f}\right) \quad \forall i \in I$

$$
\sum_{\substack{d^{\prime} \\ d^{\prime}<d \\ d^{\prime} \in D}} y_{i d^{\prime} f} \leq 1+M *\left(1-y_{i d f+1}\right) \quad \forall i \in I, d \in D, f \in F, f+1 \leq d
$$

$1-M *\left(1-y_{i d f+1}\right) \leq \sum_{\begin{array}{c}d^{\prime} \\ d^{\prime}<d \\ d^{\prime} \in D\end{array}} y_{i d^{\prime} f} \quad \forall i \in I, d \in D, f \in F, f+1 \leq d$

$\sum_{n \in N} \sum_{i \in I} x_{i j j^{\prime} d n} \geq v_{j j^{\prime} d} \quad \forall j, j^{\prime} \in J, d \in D$

$\sum_{n \in N} \sum_{i \in I} x_{i j j^{\prime} d n} \leq M \cdot v_{j j^{\prime} d} \quad \forall j, j^{\prime} \in J, d \in D$

$$
b_{j j^{\prime} d} \leq M \cdot v_{j j^{\prime} d} \quad \forall j, j^{\prime} \in J, d \in D
$$

$$
b_{j j^{\prime} d} \geq \sum_{n} \sum_{i} x_{i j j^{\prime} d n}-M \cdot\left(1-v_{j j^{\prime} d}\right) \quad \forall j, j^{\prime} \in J, d \in D
$$

$$
\sum_{n \in N} \sum_{j^{\prime} \in J} \sum_{j \in J}\left(l_{j j^{\prime} d}-d e_{j j^{\prime} d}\right) \cdot x_{i j j^{\prime} d n} \leq \text { upper } 1 \quad \forall i \in I, d \in D
$$




$$
\begin{aligned}
& \sum_{n \in N} \sum_{d \in D} \sum_{j^{\prime} \in J} \sum_{j \in J}\left(l_{j j^{\prime} d}-d e_{j j^{\prime} d}\right) \cdot x_{i j j^{\prime} d n} \leq u p p e r 2 \quad \forall i \in I \\
& 2 . z_{i j j^{\prime} j^{\prime \prime} d n} \geq x_{i j j^{\prime} d n}+x_{i j^{\prime} j^{\prime \prime} d, n+1} \quad \forall i \in I, j, j^{\prime}, j^{\prime \prime} \in J, d \in d, n \in N \\
& z_{i j j^{\prime} j^{\prime \prime} d n}+1 \leq x_{i j j^{\prime} d n}+x_{i j^{\prime} j^{\prime \prime} d, n+1} \quad \forall i \in I, j, j^{\prime}, j^{\prime \prime} \in J, d \in d, n \in N \\
& \sum_{n \in N} \sum_{j^{\prime} \in J} \sum_{j \in J}\left(l_{j j^{\prime} d}-d e_{j j^{\prime} d}\right) \cdot x_{i j j^{\prime} d n}+\sum_{n \in N} \sum_{j \in J} \sum_{j^{\prime} \in J} \sum_{j^{\prime \prime} \in J}\left(\left(d e_{j^{\prime} j^{\prime \prime} d}-l_{j j^{\prime} d}\right) \cdot z_{i j j^{\prime} j^{\prime \prime} d n}\right) \\
& \leq u p p e r 3 \quad \forall i \in I, d \in d
\end{aligned}
$$

Objective function (1) tends to minimize the total cost of the system. Total cost includes uncovered flights cost, deadhead cost, hoteling cost, and crew cost. Constraint (2) ensures that each flight can be done in a single flight round or uncovered. Constraint (3) ensures that each crew member can handle at most one flight in a flight round. Relation (4) is about minimum sit time between two consecutive flights on a duty. Constraint (5) is about minimum rest time between two consecutive flights on consecutive days. Relation (6) indicates that each crew member must start his/her first duty from home base. Constraint (7) ensures that each crew member must come back to home base at the end of his/her last duty. Constraints (8) and (9) ensure the integrity of consecutive duties. It means that if a duty ends to city $\mathrm{j}$, the next duty must start from j. Constraint (10) ensures flight integrity on a duty day. Relations (11) and (12) show that no flight should be assigned to a crew member on his/her nonworking day. Constraints (13) to (15) illustrate the relationship among working days and each of the scheduling days over the studied horizon. Constraint (16) implies that if a duty day is assigned to the crew member $\mathrm{i}$, crew cost should be considered for him/her. Constraints (17) and (18) imply that a crew member can handle a flight originating from city $\mathrm{j}$ on his/her first duty if he/she has previously arrived at $\mathrm{j}$ on that day. Constraints (19) and (20) ensure that a crew member can handle a flight originating from city $\mathrm{j}$ if he/she has previously arrived at $\mathrm{j}$ on that day or at the end of his/her previous duty. Constraint (21) states that each crew member must start his/her first flight from his/her home base. Constraint (22) ensures the integrity between two consecutive duties. Constraint (23) demonstrates all working and nonworking days of a crew member in the planning horizon. Constraint (24) implies that if a duty is not assigned to the crew member, he/she is not used in the planning horizon. Note that one of the advantages of this model is that it determines the best number of assigned crew members. So, the result of the model may indicate that some of the existing crew members need to be idle in the planning horizon. Constraints (25) and (26) show the relationship between two consecutive duties of a crew member. Constraints (27) and (28) show that if no crew member is dedicated to a flight, it will be left uncovered. Constraints (29) and (30) show deadhead flights. Constraints (31) and (32) limit the total flying time per duty and planning horizon, respectively. Constraint (35) limits the total elapsed time per duty.

\section{PROBLEM SOLVING APPROACH}

As CSP is an NP-hard problem, it is not possible to achieve an optimal solution for large-scale problem. As a result, two metaheuristic-based approaches, namely, Particle Swarm Optimization (PSO) and Genetic Algorithms (GA), have been used to find a near-optimal solution over a reasonable time. In this section, the applied metaheuristic algorithms are presented.

\subsection{Genetic algorithm}

GA is the most well-known type of evolutionary algorithms developed by Holland 1992. This algorithm is population-based, and the initial solutions are produced through the proper encoding of the problem. Then, each of the solutions is evaluated using a fitness function. In the next step, some solutions (parents) are selected through the Roulette Wheel Selection mechanism and create the Mating Pool. Subsequently, off-springs are generated through 
crossover and mutation of parents. By adding the off-springs to the set of solutions, the possibility of reaching better solutions increases. This procedure continues as long as the termination conditions are prepared.

\subsubsection{Chromosome encoding}

In this study, all possible pairings are created according to the existing crew base with two and four flights. It should be noted that all the FAA rules are considered during creation. Figure 1 shows the chromosome encoding that is used to represent the problem solutions. In this paper, this chromosome is a row vector that its columns are equal to the number of available flights (gens). These columns are sorted in a descending order based on the number of pairings related to flights. In other words, the first columns show the flights with less pairings, and the last ones show the flights with more pairings. Each column is filled by a random key number. Random keys were introduced by Been (1994) for the first time. The numbers have the uniform distribution between 0 and 1 . The flight selection starts from the beginning of this vector and continues till the end.

After selecting the flight, the pairing that contains that flight is determined through the chromosome. In fact, the random number in this chromosome is multiplied by the number of pairings related to the selected flight, and the desirable pairing is determined. The above-mentioned procedure is repeated for other selections. During flight selections, if a flight was selected by a pairing(s) before, it will be neglected. The selection procedure continues until no flight is unselected. In this study, a multipoint cross-over operator is used, and the mechanism of mutation is to select a number of genes randomly and replace them with new random numbers.

\begin{tabular}{c|c|c|c|c|c|c|c|c|c|} 
& \multicolumn{1}{c}{ flight 1 flight 2 flight 3 } & \multicolumn{1}{c}{... } & \multicolumn{4}{c}{ flight $\mathrm{n}-1$ flight $\mathrm{n}$} \\
choromose & 0.53 & 0.43 & 0.76 & 0.01 & 0.32 & 0.87 & 0.12 & 0.25 & 0.74 \\
\cline { 2 - 10 }
\end{tabular}

Figure 1. Problem encoding.

The pseudocode of the proposed GA algorithm is expressed in Figure 2.

\section{Begin.}

Initialize population with random candidate solutions

Evaluate each chromosome according to the fitness function

Repeat until the termination condition is satisfied

Select parents

Apply cross-over operator

Apply mutation operator

Evaluated new candidates

Select individuals for the next generation

End

Figure 2. Pseudocode of GA.

\subsection{Particle swarm optimization}

PSO algorithm is an evolutionary algorithm, which is represented based on the social behavior of birds. Kennedy and Eberhart introduced this algorithm in 1995. This algorithm is a population-based algorithm, and the change in each individual's position (particle) is based on its previous movements and the experience of neighboring particles. If a particle can get better situations in these replacements, these experiences will be stored in the particle's memory and 
will affect the next changes in the position of the particle. This process continues to the point that achieving a better solution is not possible. Consider that the Swarm size is equal to $\mathrm{N}$, and the position related to each of the particles in the repetition $t$ is in the form of $X_{i}(t)=\left(x_{i 1}(t), x_{i 2}(t), \ldots, x_{i D}(t)\right), i=\{1,2, \ldots, N\}$. For each solution, the vector called the velocity vector is attributed to $V_{i}(t)=\left(v_{i 1}(t), v_{i 2}(t), \ldots, v_{i D}(t)\right)$. The best solution obtained from the specified swarm until $t$ repetition is in the form of $P_{i}(t)=\left(p_{i 1}(t), p_{i 2}(t), \ldots, P_{i D}(t)\right)$, and the best solution obtained from the particles, until this repetition, is $P_{g}(t)=\left(p_{g 1}(t), p_{g 2}(t), \ldots, p_{g D}(t)\right)$ (Eddaly et al., 2016). The velocity vector and position of each swarm, in the repetition of $t+1$, are adjustable according to the following formulas.

$$
\begin{aligned}
& v_{i j}(t+1)=w \cdot v_{i j}(t)+c_{1} \cdot r_{1} \cdot\left(p_{i j}(t)-x_{i j}(t)\right)+c_{2} \cdot r_{2} \cdot\left(p_{g j}(t)-x_{i j}(t)\right) \\
& x_{i j}(t+1)=x_{i j}(t)+v_{i j}(t+1)
\end{aligned}
$$

where $c_{1}$ is cognition learning factor, $c_{2}$ social learning factor, and, $r_{1}, r_{2}$ random numbers with uniform probability distribution between 0 and $1 . w$ is the inertia weight parameter that affects the previous velocity of particle on its current velocity.

Pseudocode of the proposed PSO algorithm is expressed in Figure 3.

\section{Begin}

Initialize population with random candidate solutions

Evaluate each chromosome according to the fitness function

Step 1: Define the search space, population size, and objective function.

Step 2: Initialize a swarm of particles with random positions and velocities in the problem space.

Step 3: Define the local solution and the global best solution.

Step 4: Update the current positions and velocities using Eq (36) and Eq (37)

Step 5: Calculate the value of the objective function for each particle and update the current local best and global best.

Step 6: Go back to step (4) until a stop criterion is satisfied, usually a sufficiently good fitness or a specified number of iterations.

\section{Figure 3. Pseudocode of PSO.}

\subsection{Parameters setting}

The efficiency of the proposed algorithms considerably depends on the applied parameters. Taguchi first presented the parameter design in early 1960s. The main GA parameters/factors are maximum iteration, initial population, crossover rate, and mutation rate, while in PSO maximum iteration, Swarm size, the inertia weight $(w)$, cognitive learning factor $\left(c_{1}\right)$, and social learning factor $\left(c_{2}\right)$ are considered as the main parameters. Each of the parameters mentioned above is valued in 3 levels. For GA, the maximum iteration ranged from 80 to 120, with rises equal to 20 units. Also, the initial population ranged from 100 to 200, with changes equal to 50 units. The cross-over rate is in the range of 0.5 to 0.7 , with 0.1 -unit increases, and mutation rate is in the range of 0.1 to 0.3 , with 0.1 -unit increases. In the PSO algorithm, the maximum iteration parameter ranges from 60 to 100, with 20-unit changes. The Swarm size is in the range of 100 to 180 , with 40 -unit rises. The inertia weight $(w)$ ranges from 0.5 to 0.7 , with 0.1 -unit changes. The $c_{1}$ factor is in the range of 1.9 to 2.1, with 0.1-unit changes, and $c_{2}$ is in the range of 1.9 to 2.1, with 0.1-unit changes. For GA, 9 different scenarios are considered, while 27 different scenarios are assumed for PSO. Each scenario is repeated five times, and the mean S/N ratio plots for the GA and the PSO objective functions are depicted in Figs 4 and 5. Finally, the optimal values of these parameters are obtained as follows:

GA Parameters: $\max$ iteration $=120$, Population size $=200$, cross-over rate $=0.6$ and mutation rate $=0.3$

PSO Parameters: $\max$ iteration $=100$, swarm size $=180, w=0.7, c_{1}=2.1$ and $c_{2}=1.9$ 


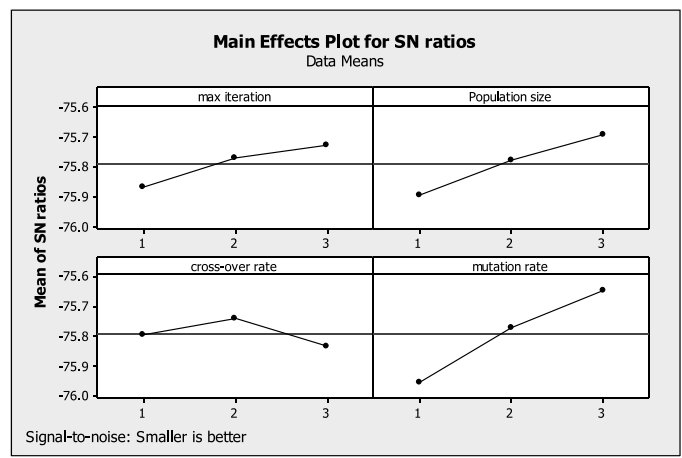

Figure 4. Mean $\mathrm{S} / \mathrm{N}$ ratio plot for each level of factors in GA.

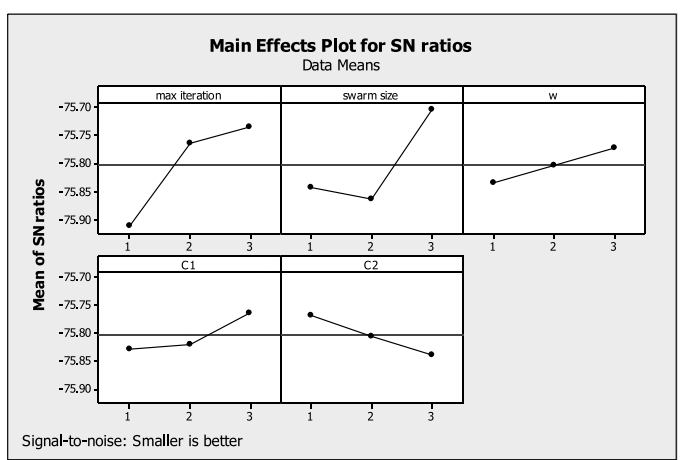

Figure 5. Mean S/N ratio plot for each level of factors in PSO.

\section{COMPUTATIONAL RESULTS}

In this paper, a set of problem instances extracted by Kasirzadeh et al. (2017) is used to validate the suggested model and evaluate the efficiency of proposed algorithms. 12 instances with different sizes are used to validate the model. Table 1 shows the details of these problems, as well as computational results. In this table, the 2 nd to 4 th columns indicate the characteristics of each problem, including the number of available flights, the number of planning horizon time, and the number of available crew. Columns 5 and 6 indicate the number of covered flights from the available set of flights, and the number of crew members used amongst the existing crew members. According to the results, metaheuristic algorithms were able to find the global solutions for small-sized problems. By increasing the problem size, the computational time of the exact approach increased considerably, so the software was stopped after a predetermined time ( 24 hours), and the solutions were recorded as local solutions. In these problems, both metaheuristic algorithms obtained the acceptable solutions (within $9 \%$ gap) significantly faster than GAMS. This shows the efficiency of the algorithms. Eventually, for large-sized problems, only the solutions obtained by the proposed algorithms are recorded. It should be noted that the comparison of the algorithms reveals that the PSO algorithm is a bit less time consuming and achieved better solutions compared to GA (Figures 6 and 7). The solutions are achieved on the laptop with the specifications of corei7, 2.4GHz, and 4GB of RAM.

Table1. computational results.

\begin{tabular}{|c|c|c|c|c|c|c|c|c|c|c|c|c|c|c|c|c|}
\hline \multirow[b]{2}{*}{ Instance } & \multicolumn{5}{|c|}{ Problem data } & \multicolumn{3}{|c|}{ GAMS } & \multicolumn{4}{|c|}{ PSO } & \multicolumn{4}{|c|}{ GA } \\
\hline & $\begin{array}{l}\text { No. of } \\
\text { flights }\end{array}$ & $\begin{array}{l}\text { No. of } \\
\text { days }\end{array}$ & $\begin{array}{l}\text { No. of } \\
\text { crew } \\
\text { members }\end{array}$ & $\begin{array}{l}\text { No. of } \\
\text { covered } \\
\text { flights }\end{array}$ & $\begin{array}{l}\text { No. of } \\
\text { used crew } \\
\text { members }\end{array}$ & $\begin{array}{l}\text { GAMS } \\
\text { solution }\end{array}$ & $\begin{array}{c}\text { GASM } \\
\text { CPU } \\
\text { time }\end{array}$ & Status & $\begin{array}{c}\text { Best } \\
\text { solution }\end{array}$ & $\begin{array}{c}\text { Ave. } \\
\text { solution }\end{array}$ & $\begin{array}{l}\text { Ave. } \\
\text { CPU } \\
\text { time }\end{array}$ & GAP $\%$ & $\begin{array}{c}\text { Best } \\
\text { solution }\end{array}$ & $\begin{array}{c}\text { Ave. } \\
\text { solution }\end{array}$ & $\begin{array}{l}\text { Ave. } \\
\text { CPU } \\
\text { time }\end{array}$ & $\mathrm{GAP} \%$ \\
\hline 1 & 35 & 2 & 15 & 35 & 10 & 3050 & 6 & Optimal & 3050 & 3070 & 7 & 0.00 & 3050 & 3070 & 7 & 0.00 \\
\hline 2 & 38 & 2 & 15 & 38 & 11 & 3390 & 40 & Optimal & 3390 & 3501 & 20 & 0.00 & 3390 & 3690 & 29 & 0.00 \\
\hline 3 & 50 & 2 & 20 & 50 & 14 & 4280 & 33 & Optimal & 4280 & 4300 & 35 & 0.00 & 4280 & 4340 & 37 & 0.00 \\
\hline 4 & 26 & 3 & 15 & 26 & 8 & 2520 & 13 & Optimal & 2520 & 2520 & 6 & 0.00 & 2520 & 2540 & 10 & 0.00 \\
\hline 5 & 31 & 3 & 15 & 31 & 10 & 3230 & 420 & Optimal & 3230 & 3230 & 42 & 0.00 & 3300 & 3396 & 51 & 2.17 \\
\hline 6 & 31 & 3 & 15 & 31 & 9 & 2870 & 10 & Optimal & 2870 & 2870 & 11 & 0.00 & 2870 & 2901 & 15 & 0.00 \\
\hline 7 & 26 & 3 & 15 & 26 & 9 & 2880 & 23 & Optimal & 2880 & 2880 & 7 & 0.00 & 2880 & 2922 & 10 & 0.00 \\
\hline 8 & 70 & 4 & 30 & 70 & 19 & 5800 & $\begin{array}{l}21 \text { hour } \\
\text { and } 10 \\
\min , 32 \\
\text { sec }\end{array}$ & Optimal & 6170 & 6310 & $\begin{array}{l}19 \mathrm{~min} \\
\text { and } \\
32 \mathrm{sec}\end{array}$ & 6.38 & 6330 & 6425 & $\begin{array}{c}23 \mathrm{~min} \\
\text { and } 2 \\
\text { sec }\end{array}$ & 9.14 \\
\hline 9 & 76 & 4 & 30 & 76 & 23 & 7080 & 24 hour & Local & 7240 & 7354 & $\begin{array}{c}15 \mathrm{~min} \\
\text { and } 19 \\
\text { sec }\end{array}$ & 2.26 & 7310 & 7421 & $\begin{array}{c}17 \mathrm{~min} \\
\text { and } 48 \\
\text { sec }\end{array}$ & 3.25 \\
\hline 10 & 57 & 5 & 30 & 57 & 21 & 5270 & 24 hour & Local & 5450 & 5596 & $\begin{array}{c}25 \mathrm{~min} \\
\text { and } 29 \\
\mathrm{sec}\end{array}$ & 3.42 & 5510 & 5798 & $\begin{array}{c}26 \mathrm{~min} \\
\text { and } 38 \\
\mathrm{sec}\end{array}$ & 4.55 \\
\hline 11 & 93 & 6 & 40 & - & - & - & - & - & 15423 & 16012 & $\begin{array}{c}30 \mathrm{~min} \\
\text { and } 32 \\
\mathrm{sec}\end{array}$ & - & 16190 & 17011 & $\begin{array}{c}32 \mathrm{~min} \\
\text { and } 4 \\
\sec \end{array}$ & - \\
\hline 12 & 134 & 6 & 50 & - & - & - & - & - & 14960 & 15569 & $\begin{array}{c}34 \mathrm{~min} \\
\text { and } 4 \\
\text { sec }\end{array}$ & - & 15645 & 16901 & $\begin{array}{c}38 \mathrm{~min} \\
\text { and } 51 \\
\text { sec }\end{array}$ & - \\
\hline
\end{tabular}




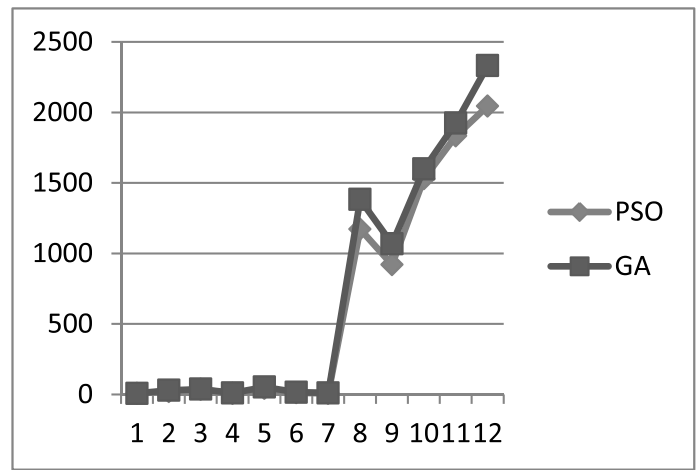

Figure 6. Comparison of problem-solving time between PSO and GA.

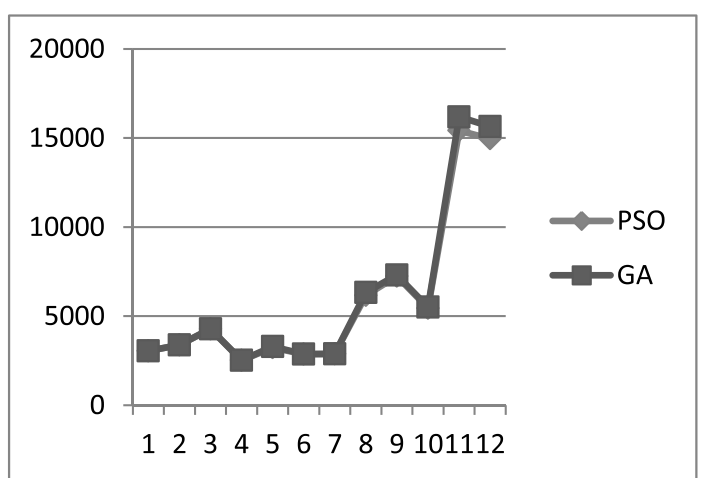

Figure 7. Comparison of the achieved solutions between PSO and GA.

\section{DISCUSSION}

In this section, the performance of the proposed integrated mathematical model for CSP is evaluated. First, two separate mathematical formulations for the CPP and CRP are presented. Then, two models are solved based on the sequential order so that the CPP outputs are assumed as CRP inputs, and the final results are achieved by solving the CRP model. At the end, the achieved results can be compared with integrated ones in terms of objective functions and CPU time.

\section{CPP formulation:}

For the CPP formulation, the following modifications in CSP integrated model are required. New Sets, indices, and constraints for this problem are as follows:

\section{Sets and indices}

$P$ : Set of all pairings

$p:$ Index of pairing $(\mathrm{p} \in P)$

$O$ : Set of all home base

$o:$ Index of home base $(o \in O)$

\section{Constraints}

$$
\begin{aligned}
& \sum_{j \in O} s_{p j d} \geq-M *\left(1-y_{p d f}\right)+1 \quad \forall p \in P, f \in F, f=1 \\
& w_{p j d} \geq s_{p j d^{\prime \prime}}-M *\left(1-y_{p d f}\right)-M *\left(\sum_{\substack{d^{\prime}=d+1 \\
d^{\prime} \in D}} \sum_{\substack{f^{\prime}=f+1 \\
f^{\prime} \in F}} y_{p d^{\prime} f^{\prime}}\right)-M *\left(1-y_{p d^{\prime \prime} f^{\prime \prime}}\right) \\
& \forall p \in P, j \in O, d \in D, f, f^{\prime \prime} \in F: f \leq d, f^{\prime \prime} \leq d^{\prime \prime}, \quad f^{\prime \prime}=1
\end{aligned}
$$

An index of $i$ is replaced with $p$ in the formulations presented in Section 4, and the new index of $o$ is also introduced. The constraints (6), (7), and (16) are removed from the model, and the new constraints (38) and (39) are added. The new constraints ensure that each pairing must start from and end at the same home base. Finally, the crew cost item in the objective function is also removed. 


\section{CRP formulation:}

Sets, indices, parameters, and decision variables for the problem of CRP are as follows.

\section{Sets and indices}

I: Set of all crew members

$i$ : Index of crew member $(i \in I)$

$P$ : Set of all pairings

$p:$ Index of pairing $(\mathrm{p} \in P)$

$o_{p}:$ Home base of pairing $\mathrm{p}$

\section{Parameter}

$r_{i}$ : cost of using crew i.

\section{Decision variables}

$u_{i p}$ : If pairing $\mathrm{p}$ is assigned to crew member $\mathrm{i}, 1$ otherwise 0

\section{Constraints and objective function}

$$
\begin{aligned}
& \sum_{i \in o_{p}} u_{i p}=1 \quad \forall p \in P \\
& \sum_{p \in o_{i}} u_{i p} \leq 1 \quad \forall i \in i \\
& \min \sum_{i \in I} \sum_{\mathrm{p} \in P} u_{i p} * r_{i}
\end{aligned}
$$

Constraint (40) states that each selective pairing must be assigned to only one of the crew members with the same home base. Constraint (41) ensures that each crew member can handle at most one selective pairing. The objective function (42) tends to minimize the total cost of the system including crew cost assignment.

Finally, the results of the proposed combined model are compared with the results obtained when the two models are considered separately through some test problems (Table 2). As can be seen, in contrast to the sequential models, the integrated one generates accurate and appropriate work schedules for the existing crew members, leading to $14.39 \%$ average cost reduction in only $7.27 \%$ CPU time increase. The archived results show the superiority of the integrated model in comparison to the sequential order ones. 
Table 2. Comparison between the integrated model and sequential order ones.

\begin{tabular}{|c|c|c|c|c|c|c|}
\hline \multirow{2}{*}{ Instance } & \multicolumn{2}{|c|}{ Integrated model } & \multicolumn{2}{|c|}{ Sequential order models } & \multirow{2}{*}{$\begin{array}{c}\text { Related } \\
\text { percentage of } \\
\text { deviation (RPD) } \\
\text { objective } \%\end{array}$} & \multirow{2}{*}{$\begin{array}{c}\text { Related } \\
\text { percentage of } \\
\text { deviation (RPD) } \\
\text { CPU time } \%\end{array}$} \\
\hline & $\begin{array}{l}\text { Objective } \\
\text { function }\end{array}$ & $\begin{array}{c}\mathrm{CPU} \\
\text { time(se) }\end{array}$ & $\begin{array}{l}\text { Objective } \\
\text { function }\end{array}$ & $\begin{array}{c}\mathrm{CPU} \\
\text { time(sec) }\end{array}$ & & \\
\hline 1 & 4280 & 33 & 4731 & 31 & 10.54 & 6.45 \\
\hline 2 & 3050 & 6 & 3391 & 6 & 11.18 & 0.00 \\
\hline 3 & 3230 & 420 & 3791 & 399 & 17.37 & 5.26 \\
\hline 4 & 3390 & 40 & 3732 & 38 & 10.09 & 5.26 \\
\hline 5 & 2520 & 13 & 2822 & 12 & 11.98 & 8.33 \\
\hline 6 & 2870 & 10 & 3254 & 9 & 13.38 & 11.11 \\
\hline 7 & 2880 & 23 & 3234 & 21 & 12.29 & 9.52 \\
\hline \multirow[t]{2}{*}{8} & 5800 & 76232 & 7442 & 67938 & 28.31 & 12.21 \\
\hline & & & & Average & 14.39 & 7.27 \\
\hline
\end{tabular}

\section{CONCLUSION}

Crew scheduling problem has always been one of the researchers' interests; however, due to the complex nature of this problem, it has always been solved in two separate steps, namely, crew pairing and crew rostering problems. It should be noted that few investigations have been concerned on integrating these two steps. In this study, a new integrated mathematical model of the two above steps was introduced considering the rules of aviation. Due to the Np-hardness of the problem, it was not possible to achieve an optimal solution for large-sized problem over a reasonable amount of time. Therefore, two solution approaches, namely, particle swarm optimization (PSO) and genetic algorithm (GA), were implemented. In the case of the small-sized problems, it was concluded that both of the algorithms were able to achieve an optimal solution. By increasing the problem size, the software computational time was highly increased; consequently, metaheuristic algorithms were used for large-sized problem and could achieve the acceptable solution in a reasonable time. When it comes to the comparison of the algorithms in terms of optimality of the solutions and computing time, PSO outperformed GA, which in turn indicates its successful performance. Moreover, the results of the proposed combined model were compared with the results obtained when the two models are considered separately through some test problems. In comparison to the sequential order models, the proposed integrated model in this study generated more accurate and appropriate work schedules for the existing crew members, leading to about $14.39 \%$ average cost reduction in only $7.27 \%$ CPU time increase. It is suggested that researchers investigate the problem by considering the possibility of having more than one flight from a specific origin to a specific destination daily. Consideration of other aviation laws relating to duties and vacations is also suggested.

\section{REFERENCES}

AhmadBeygi, S., Cohn, A. \& Weir, M. 2009. An integer programming approach to generating airline crew pairings. Computers \& Operations Research, 36(4): 1284-1298.

Ahmed, M.B., Mansour, F.Z. \& Haouari, M. 2018. Robust integrated maintenance aircraft routing and crew pairing. Journal of Air Transport Management, 73(1): 15-31.

Aydemir-Karadag, A., Dengiz, B. \& Bolat, A. 2013. Crew pairing optimization based on hybrid approaches. Computers \& Industrial Engineering, 65(1): 87-96. 
Azadeh, A., Farahani, M.H., Eivazy, H., Nazari-Shirkouhi, S. \& Asadipour, G. 2013. A hybrid meta-heuristic algorithm for optimization of crew scheduling. Applied Soft Computing, 13(1): 158-164.

Barnhart, C. \& Talluri, K.T. 1997. Airline operations research. Design and operation of civil and environmental engineering systems, 435-469.

Bazargan, M. 2016. Airline operations and scheduling. Routledge.

Bean, J.C. 1994. Genetic algorithms and random keys for sequencing and optimization. ORSA journal on computing, 6(2): 154-160.

Cacchiani, V. \& Salazar-Gonzalez, J.J. 2013. A heuristic approach for an integrated fleet-assignment, aircraft-routing and crewpairing problem. Electronic Notes in Discrete Mathematics, 41(1): 391-398.

Chen, C.H. \& Chou, J.H. 2016. Multiobjective optimization of airline crew roster recovery problems under disruption conditions. IEEE Transactions on Systems, Man, and Cybernetics: Systems, 47(1): 133-144.

Cordeau, J.F., Stojkovic, G., Soumis, F. \& Desrosiers, J. 2001. Benders decomposition for simultaneous aircraft routing and crew scheduling. Transportation science, 35(4): 375-388.

De Armas, J., Cadarso, L., Juan, A.A. \& Faulin, J. 2017. A multi-start randomized heuristic for real-life crew rostering problems in airlines with work-balancing goals. Annals of Operations Research, 258(2): 825-848.

Deng, G.F. \& Lin, W.T. 2011. Ant colony optimization-based algorithm for airline crew scheduling problem. Expert Systems with Applications, 38(5): 5787-5793.

Deveci, M. \& Demirel, N.C. 2018. Evolutionary algorithms for solving the airline crew pairing problem. Computers \& Industrial Engineering, 115(1): 389-406.

Díaz-Ramírez, J., Huertas, J.I. \& Trigos, F. 2014. Aircraft maintenance, routing, and crew scheduling planning for airlines with a single fleet and a single maintenance and crew base. Computers \& Industrial Engineering, 75(1): 68-78.

Doi, T., Nishi, T. \& Vob, S. 2018. Two-level decomposition-based matheuristic for airline crew rostering problems with fair working time. European Journal of Operational Research, 267(2): 428-438.

Dong, Z., Chuhang, Y. \& Lau, H.H. 2016. An integrated flight scheduling and fleet assignment method based on a discrete choice model. Computers \& Industrial Engineering, 98(1): 195-210.

Eddaly, M., Jarboui, B. \& Siarry, P. 2016. Combinatorial particle swarm optimization for solving blocking flowshop scheduling problem. Journal of Computational Design and Engineering, 3(4): 295-311.

Gao, C., Johnson, E. \& Smith, B. 2009. Integrated airline fleet and crew robust planning. Transportation Science, 43(1): 2-16.

Gomes, W.P. \& Gualda, N.D. 2015. Heuristics to solve the integrated airline crew assignment problem. Journal of Transport Literature, 9(1): 25-29.

Graves, G.W., McBride, R.D., Gershkoff, I., Anderson, D. \& Mahidhara, D. 1993. Flight crew scheduling. Management science, 39(6): 736-745.

Hadianti, R., Novianingsih, K., Uttunggadewa, S., Sidarto, K.A., Sumarti, N. \& Soewono, E. 2013. Optimization model for an airline crew rostering problem: Case of Garuda Indonesia. Journal of Mathematical and Fundamental Sciences, 45(3): 218-234.

Haouari, M., Shao, S. \& Sherali, H.D. 2013. A lifted compact formulation for the daily aircraft maintenance routing problem. Transportation Science, 47(4): 508-525.

Haouari, M., Zeghal Mansour, F. \& Sherali, H.D. 2019. A New Compact Formulation for the Daily Crew Pairing Problem. Transportation Science, 53(3): 811-828.

Holland, J.H. 1992. Adaptation in natural and artificial systems: an introductory analysis with applications to biology, control, and artificial intelligence. MIT press.

Ionescu, L. \& Kliewer, N. 2011. Increasing flexibility of airline crew schedules. Procedia-Social and Behavioral Sciences, 20(1): 1019-1028.

Kasirzadeh, A., Saddoune, M. \& Soumis, F. 2017. Airline crew scheduling: models, algorithms, and data sets. EURO Journal on 
Transportation and Logistics, 6(2): 111-137.

Kennedy, J. \& Eberhart, R. 1995. Particle swarm optimization. In Proceedings of ICNN'95-International Conference on Neural Networks, 4(1): 1942-1948.

Kohl, N. \& Karisch, S.E. 2004. Airline crew rostering: Problem types, modeling, and optimization. Annals of Operations Research, 127(1): 223-257.

Mercier, A. \& Soumis, F. 2007. An integrated aircraft routing, crew scheduling and flight retiming model. Computers \& Operations Research, 34(8): 2251-2265.

Mercier, A., Cordeau, J.F. \& Soumis, F. 2005. A computational study of Benders decomposition for the integrated aircraft routing and crew scheduling problem. Computers \& Operations Research, 32(6): 1451-1476.

Muter, İ., Birbil, Ş.İ., Bulbul, K., Sahin, G., Yenigun, H., Tas, D. \& Tuzun, D. 2013. Solving a robust airline crew pairing problem with column generation. Computers \& Operations Research, 40(3): 815-830.

Ozdemir, Y.S. and Uskudar, A., 2020. Strategy selection by using interval type-2 fuzzy mcdm and an application. Journal of Engineering Research, 8(3).

Ozener, O.O., Matoglu, M.O., Erdogan, G., Haouari, M. \& Sozer, H. 2017. Solving a large-scale integrated fleet assignment and crew pairing problem. Annals of Operations Research, 253(1): 477-500.

Quesnel, F., Desaulniers, G. \& Soumis, F. 2017. A new heuristic branching scheme for the crew pairing problem with base constraints. Computers \& Operations Research, 80(1): 159-172.

Salazar-Gonzalez, J.J. 2014. Approaches to solve the fleet-assignment, aircraft-routing, crew-pairing and crew-rostering problems of a regional carrier. Omega, 43(1): 71-82.

Sandhu, R. \& Klabjan, D. 2007. Integrated airline fleeting and crew-pairing decisions. Operations Research, 55(3): 439-456.

Santosa, B., Sunarto, A. \& Rahman, A. 2010. Using differential evolution method to solve crew rostering problem. Applied Mathematics, 1(04): 316-325.

Sohoni, M.G., Johnson, E.L. \& Bailey, T.G. 2006. Operational airline reserve crew planning. Journal of Scheduling, 9(3): 203-221.

Weide, O., Ryan, D. \& Ehrgott, M. 2010. An iterative approach to robust and integrated aircraft routing and crew scheduling. Computers \& Operations Research, 37(5): 833-844.

Zeren, B. \& Ozkol, I. 2016. A novel column generation strategy for large scale airline crew pairing problems. Expert Systems with Applications, 55(1): 133-144.

Zeren, B., \& Ozkol, İ. 2012. An improved genetic algorithm for crew pairing optimization. Journal of Intelligent Learning Systems and Applications, 4(01): 70-80. 